\title{
Über Karnin und Inosinsäure
}

\author{
(I. Mitteilung) \\ von \\ F. Haiser und F. Wenzel.
}

Aus dem Privatlaboratorium Scheibbs und dem I. chemischen Laboratorium der k. k. Universität in Wien,

(Vorgelegt in der Sitzung am 12. Dezember 1907.)

Als Karnin bezeichnete Weide $1^{1}$ eine organische Base, welche er 1871 aus Liebig's. Fleischextrakt in der folgenden Weise isolierte: Das mit Wasser verdünnte Extrakt wurde zunächst mit Barythydrat von Phosphaten etc. befreit und dann mit basischem Bleiacetat ausgefällt. Unter den ausgeschiedenen Bleiverbindungen befindet sich auch das Karninblei, welches in heiBem Wasser löslich ist und daher durch Auskochen des gewaschenen Bleiniederschlages mit Wasser von den übrigen Verbindungen getrennt werden kann. Die wässerige Lösung wird durch Einleiten von Schwefelwasserstoff und Filtrieren von Blei befreit und stark konzentriert. Es scheidet sich dann beim Erkalten das Karnin in Form eines krümlichen, noch sehr gefärbten Kristallschlammes ab, welcher nach dem Umkristallisieren mit Tierkohle in kreideweißen Drusen und krümlichen Gruppen äußerst kleiner, trikroskopischer, unregelmäßig begrenzter Kristalle ausfällt und bei weiterem Umkristallisieren die Zusammensetzung nicht ändert. Den Gehalt des Fleischextraktes an Karnin schätzt Weidel nach der Menge des erhaltenen Rohproduktes auf etwa $1 \%$.

1 Ann. der Chemie, 158, 356 (1871). 
Bald nachher wurde das Karnin von Schützenberger ${ }^{1}$ in der Hefe und von Pouchet ${ }^{2}$ im Harn aufgefunden.

1883 haben es Kruckenberg und Wagner ${ }^{3}$ unternommen, Weidel's Arbeit zu kontrollieren; sie konnten Weidel's Resultate nur bestätigen, haben sie jedoch mehrfach ergänzt, indem sie das Karnin besser charakterisierten und namentlich angaben, daß es sich bei $230^{\circ} \mathrm{zu}$ bräunen beginnt und bei $239^{\circ}$ vollständig verkohlt. Sie haben das Karnin nicht allein aus Fleischextrakt, und zwar in einer Ausbeute von 1 bis $2 g$ pro Pfund - und auch weniger - erhalten, sondern auch aus Froschfleisch und dem Fleische von Süßwasserfischen.

$\mathrm{Balke}^{4}$ konnte es aus Pferdefleisch isolieren und hat es gemeinsam mit einigen anderen Xanthinbasen zuerst in Form der Kupferoxydulverbindungen durch Anwendung von Fehling'scher Lösung und Hydroxylamin abgeschieden und nach Überführung in die Bleiverbindungen von den übrigen getrennt.

E. v. Lippmann ${ }^{5}$ endlich ist es gelungen, dasselbe unter den stickstoffhaltigen Bestandteilen des Saftes der Zuckerrübe aufzufinden.

Mit Rücksicht auf Zahl und Namen der Forscher, welche das Karnin in Händen hatten, konnte man zunächst keinesfalls daran zweifeln, daß dem Karnin die mehrfach bestätigte Formel $\mathrm{C}_{7} \mathrm{H}_{8} \mathrm{~N}_{4} \mathrm{O}_{3}$ oder doch eín Multiplum derselben tatsächlich zukommt, und da Weidel bereits beobachtet hat, daß bei der Behandlung von Karnin mit Salpetersäure oder Bromwasser Hypoxanthin (Sarkin) $\mathrm{C}_{5} \mathrm{H}_{4} \mathrm{~N}_{4} \mathrm{O}$ entsteht, mußte es als eine leichte Aufgabe erscheinen, die Kanstitution des Karnins zu ermitteln, was ja auch bereits versucht worden ist. Merkwürdigerweise aber wurde die Erreichung dieses Zieles bisher nur auf synthetischem Wege angestrebt. Schmidt und

1 Bul1. soc. chim. (2), 21, 217 (1874).

2 S. Pouchet, Contributions à la connaissance des matières extractives de l'urine. Paris, 1880.

3 Journ. für prakt. Chemie (2), 47, 553.

4 Sitzungsber. der med.-physik. Gesellsch. zu Würzburg, 1883, 58.

5 Ber. der deutschen chem. Gesellsch., 29, 2650. 
Pressler ${ }^{1}$ hielten es für möglich, daß das Karnin ein Oxytheobromin sein könnte und gingen daran, ein solches aufzubauen; sie haben aber ihre Versuche abgebrochen, um nicht mit E. Fischer, der damals in der Puringruppe arbeitete, zu kollidieren. Die Annahme, daß das Karnin ein Oxytheobromin sei, hatte allerdings wenig Wahrscheinlichkeit für sich, da, wie eben erwähnt, das Karnin durch Oxydation in das sauerstoffärmere Hypoxanthin übergeht. Weidel hat auch bereits darauf hingewiesen, daß das Karnin von Hypoxanthin sich um die Bestandteile der Essigsäure unterscheidet:

$$
\mathrm{C}_{7} \mathrm{H}_{8} \mathrm{~N}_{4} \mathrm{O}_{3}=\mathrm{C}_{5} \mathrm{H}_{4} \mathrm{~N}_{4} \mathrm{O}+\mathrm{C}_{2} \mathrm{H}_{4} \mathrm{O}_{2}
$$

und Kruckenberg und Wagner haben versucht, Hypoxanthin und Essigsäure zu vereinigen, obgleich sie es mit Rücksicht auf die Alkalibeständigkeit des Karnins für unwahrscheinlich hielten, daß dieses eine Verbindung des Hypoxanthins mit Essigsäure sein könnte.

Im Hinblick auf die im vorstehenden ausgeführte Sachlage hatten wir nun den Entschluß gefaßt, zu versuchen, die Konstitution des Karnins auf analytischem Wege aufzuklären, konnten aber doch nicht widerstehen, zunächst einen Versuch auszuführen, der uns möglicherweise leicht und rasch zum Ziele führen könnte. Wir hatten bereits festgestellt, daß das Karnin, welches nach den Angaben von Balke und nach unseren eigenen Beobachtungen Fehling'sche Lösung nicht reduziert, durch Kochen mit Salzsäure in Hypoxanthin und in einen Syrup zerfällt, der nach Entfernung des Hypoxanthins beim Erwärmen mit Fehling'scher Lösung rotes Kupferoxydul zur Ausscheidung bringt. Es schien daher, abgesehen von physiologischen Momenten, sehr leicht möglich, daß die Spaltung des Karnins durch Säureeinwirkung eine hydrolytische sei im Sinne der Gleichung:

$$
\mathrm{C}_{7} \mathrm{H}_{8} \mathrm{~N}_{4} \mathrm{O}_{3}+\mathrm{H}_{2} \mathrm{O}=\mathrm{C}_{5} \mathrm{H}_{4} \mathrm{~N}_{4} \mathrm{O}+\mathrm{C}_{2} \mathrm{H}_{6} \mathrm{O}_{3}
$$

und daß neben Hypoxanthin Glykolaldehyd zunächst in der Orthoform entsteht. Unter dieser Voraussetzung mußte das

1 Ann. der Chemie, 217, 302. 
Karnin mit Rücksicht darauf, daß es selbst nicht reduziert, optisch aktiv sein, was sich in der Tat bestätigte. Es erübrigte nun bloB, den Glykolaldehyd als solchen zu charakterisieren. Durch Kochen mit essigsaurem Phenylhydrazin wurde auch ein Osazon erhalten, welches den Schmelzpunkt $163^{\circ}$ hatte, sich jedoch mit dem einerseits aus Formaldehyd, andrerseits aus Glykolaldehyd zum Vergleiche dargestellten Glyoxalosazon keineswegs identisch erwies.

Auch an der Inosinsäure haben wir zur selben Zeit, als dies beim Karnin geschah, optische Aktivität und die Entstehung eines Osazons vom Schmelzpunkte $163^{\circ}$ konstatiert, was bei dem Umstande, daß beide Substanzen bei der Spaltung Hypoxanthin geben, eine genetische Beziehung zwischen ihnen nicht unmöglich erscheinen ließ. Wir wandten uns zunächst dem einfacheren der beiden Stoffe, dem Karnin, $z u$ und richteten in erster Linie unser Augenmerk auf die Gewinnung einer möglichst reinen Substanz.

Das Extrakt, welches wir zu unseren Versuchen verwendeten, war Liebig's Frey Bentos Fleischextrakt. In dem sogenannten Armour's Fleischextrakt aus Chicago konnten weder Inosinsäure noch Karnin auch nur in Spuren aufgefunden werden.

\section{Darstellung des Karnins.}

Die Darstellung des Karnins erfolgte zunächst ganz nach Weidel's Vorschrift. Da die Flüssigkeit nach dem Ausfällen mit Barythydrat stets stark alkaliseh und dem Geruche nach ammoniakalisch ist, so fällt mit basischem Bleiacetat neben zahireichen anderen Verbindungen sowohl Inosinsäure als auch Karnin aus. Das Bleisalz der Inosinsäure ist in heißem Wasser unlöslich, das des Karnins dagegen löslich. In früher beschriebener Weise wurde aus den löslichen Bleiverbindungen durch Schwefelwasserstoff das Blei entfernt und zur Kristallisation eingedampft. Hiebei tritt, wie Weidel bemerkt, nur manchmal Kristallisation von Karnin ein, was von den wechselnden Mengen Kochsalz abhängt, die im Fleischextrakt vorhanden sind, indem durch Vermittlung von Chlorblei in die schließlich resultierende Karninlösung verschiedene Mengen 
von Chlorwasserstoff gelangen, die zur Bildung von leicht löslichem Karninchlorhydrat Veranlassung geben. Wir konnten hier stets größere Mengen von Karnin erhalten, wenn vor dem Konzentrieren der entbleiten Lösung die Salzsäure durch Ammoniak gebunden wurde. Auch die Inosinsäure kann aus den in heißem Wasser unlöslichen Bleiverbindungen gewonnen werden; doch hat sich diese Methode der Aufarbeitung des Fleischextraktes einerseits wegen der nötigen, lang andauernden Erhitzung der unbeständigen Verbindungen auf die Siedetemperatur des Wassers, andrerseits wegen der Komplikation des Verfahrens weniger vorteilhaft erwiesen als die folgende, bei weiteren Darstellungen von uns stets benützte Arbeitsweise.

Das Extrakt wird in zirka 5 Teilen warmen Wassers von ungefähr $40^{\circ}$ gelöst und Barythydrat zugesetzt, bis kein Niederschlag erfolgt. Man scheue sich nicht davor, daß im Filtrate Baryt erscheint, sondern setze so lange Baryt $\mathrm{zu}$, als im Filtrate noch ein Niederschlag entsteht. Es wird hiebei aus so verdünnter Lösung weder Inosinsäure noch Karnin gefällt, was bei Inosinsäure wenigstens in konzentrierterer Lösung der Fall wäre. Nach dem Absaugen des Barytniederschlages, der übrigens nach dem Auswaschen mit heißem Wasser nur Spuren von organischen Substanzen enthält, wird die stark alkalische Flüssigkeit mit Essigsäure neutralisiert. Sodann wird in der Kälte mit Bleiessig ausgefällt, indem man so lange davon zusetzt, bis eben kein Niederschlag mehr entsteht. Ein Überschuß ist zu vermeiden, da dieser lösend auf den Niederschlag wirkt. Aus dem mit kaltem Wasser gut ausgewaschenen Niederschlage gewinnt man nach Zerlegung mit Schwefelwasserstoff in der Kälte, Aufkochen mit Baryumcarbonat und Einkochen des Filtrates im Vakuum den inosinsauren Baryt, und zwar etwa 5 bis $6 g$ aus einem Pfund Fleischextrakt. Die Ausbeute hängt ganz von der Frische desselben ab; aus alten dunkelbraunen Sorten wurde oft nicht die Spur gewonnen, aus ganz frischen hellgelben dagegen $7 g$.

Das Filtrat von der Bleiessigfällung wird mit Ammoniak versetzt, wobei abermals ein Bleiniederschlag entsteht, der das Karnin enthält. Der Niederschlag wird abfiltriert, mit kaltem 
Wasser gewaschen und mit Schwefelwasserstoff zerlegt. Dies kann in der Hitze vorgenommen werden, geschieht jedoch zweckmäßiger auch in der Kälte, damit etwa entstandene freie Säuren keine Zersetzung hervorrufen. Noch vor dem Abfiltrieren des Bleisulfids werden die Säuren mit Baryumcarbonat neutralisiert, hierauf wird filtriert und zum Sirup eingedampft. Nach dem Impfen mit Karninkristallen oder nach 24stündigem Stehen kommt die ganze Masse zur Kristallisation. Die Kristalle werden abgesaugt, kalt gewaschen und drei- bis viermal unter Eindampfen mit wenig Tierkohle aus Wasser umkristallisiert. Die Ausbeute beträgt ebenfalls 5 bis $6 g$ pro Pfund frischen Fleischextraktes.

\section{Eigenschaften des Karnins.}

Das so gewonnene Karnin gleicht ganz und völlig der Beschreibung nach dem Weidel'schen Präparat. Es ist ein krümlicher Kristallschlamm, erscheint nach dem Trocknen kreidig und glanzlos und färbt sich entsprechend den Angaben von Kruckenberg und Wagner bei $230^{\circ}$ braun, um bei höherer Temperatur zu verkohlen. Es ist in kaltem Wasser sehr schwer, in heißem leichter löslich, in Alkohol und in Äther ist es nahezu unlöslich. Der Bleiessigniederschlag ist in der Hitze löslich in Wasser, was übrigens auch von dem des Hypoxanthins gilt. Der voluminöse Silberniederschlag scheint in Ammoniak unlöslich; tatsächlich ist ein Teil desselben ammoniaklöslich, wie im späteren angeführt werden wird.

Dieses Karnin wurde weiterhin 14 mal umkristallisiert und nach je zwei bis drei Kristallisationen analysiert. Hiebei ergaben sich Werte, die den von der Formel $\mathrm{C}_{7} \mathrm{H}_{8} \mathrm{~N}_{4} \mathrm{O}_{3}$ geforderten sich einigermaßen nähern:

In 100 Teilen:

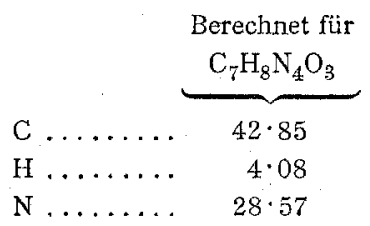

\begin{tabular}{rrrr}
\multicolumn{4}{c}{ Gefunden } \\
\multicolumn{1}{c}{ I. } & \multicolumn{1}{c}{ II. } & \multicolumn{1}{c}{ III. } & \multicolumn{1}{c}{ IV. } \\
$44 \cdot 04$ & $43 \cdot 98$ & $44 \cdot 80$ & $44 \cdot 14$ \\
$4 \cdot 37$ & $3 \cdot 86$ & $3 \cdot 23$ & $3 \cdot 90$ \\
$27 \cdot 71$ & $28 \cdot 40$ & $28 \cdot 99$ & $27 \cdot 70$
\end{tabular}


Da die Differenzen in den aus den Analysen berechneten Prozentzahlen weit über die Analysenfehler hinausgehen und durch weiteres Umkristallisieren die Erreichung einer vollständigen Konstanz der Werte nicht zu erwarten war, gingen wir daran, die Reinigung der Substanz auf anderen Wegen zu versuchen und wollten zunächst das Chlorhydrat hiefür benützen.

Proben von zwei Kristallisationen, die starke Abweichung voneinander im Stickstoffgehalte zeigten, wurden in heißer, verdünnter Salzsäure gelöst, das ausgeschiedene Chlorhydrat umkristallisiert und die Base durch Ammoniak wieder in Freiheit gesetzt. Es zeigte sich jedoch bald, daß bei dieser Behandlung reines Hypoxanthin entstanden war. Die Schwerlöslichkeit in Wasser hatte zugenommen, beim Erhitzen trat bei $230^{\circ}$ keine Braunfärbung mehr ein und auch sonst zeigte die Substanz alle Eigenschaften des reinen Hypoxanthins. Die Analyse der aus jeder der beiden Proben erhaltenen Base ergab die folgenden, gleichfalls auf Hypoxanthin stimmenden Zahlen:

I. $0 \cdot 1357 \mathrm{~g}$ Substanz gaben $51.0 \mathrm{~cm}^{3}$ feuchten Stickstoff bei $23^{\circ}$ und $739 \mathrm{~mm}$ Barometerstand.

II. $0 \cdot 1051 \mathrm{~g}$ Substanz gaben $39 \cdot 6 \mathrm{~cm}^{3}$ feuchten Stickstoff bei $22^{\circ}$ und 736 mm Barometerstand.

In 100 Teilen:

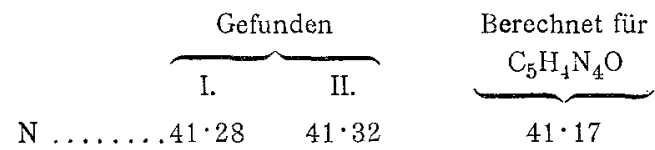

Da wir auf diese Weise zum Chlorhydrat des Karnins nicht gelangt waren, dasselbe aber mehrfach beschrieben ist, haben wir, um die Wirkung der Salzsäure möglichst abzuschwächen, $1 / 2 g$ Karnin in der berechneten Menge Normalsalzsäure bei einer Temperatur von $50^{\circ}$ gelöst und die Flüssigkeit ohne weitere Erwärmung im Vakuum eingedunstet. Von der aus dem Chlorhydrat durch Ammoniak zurückgewonnenen Base wurde eine Stickstoffbestimmung gemacht mit dem folgenden Ergebnisse: 
$0.0999 \mathrm{~g}$ Substanz gaben $29.0 \mathrm{~cm}^{3}$ feuchten Stickstoff bei $22^{\circ}$ und $740 \mathrm{~mm}$ Barometerstand.

In 100 Teilen:

\begin{tabular}{|c|c|c|c|}
\hline & \multirow{2}{*}{ Gefunden } & \multicolumn{2}{|c|}{ Berechnet für } \\
\hline & & Karnin & Hypoxanthin \\
\hline$\ldots \ldots$ & $32 \cdot 14$ & $28 \cdot 57$ & $41 \cdot 17$ \\
\hline
\end{tabular}

Es hat also diese äußerst vorsichtige Behandlung des Karnins mit Salzsäure auch bereits ein Steigen des Stickstoffgehaltes verursacht. Die gleiche Empfindlichkeit zeigte das Karnin auch bei dem Versuche zur Darstellung eines Goldsalzes; selbst bei der größten Vorsicht konnte nur das

\section{Hypoxanthin-Goldchlorid}

erhalten werden. Es wurde bei einem Versuche das Karnin unter Eiskühlung in Salzsäure gelöst, Goldchlorid zugesetzt und die Lösung im Vakuum über Schwefelsäure verdunsten gelassen. Das Golddoppelsalz schied sich in Form wohlausgebildeter, glänzender Prismen und Nadeln aus, die in Wasser ziemlich schwer löslich waren. Die Analyse derselben ergab:

I. $0 \cdot 1765 g$ Substanz (bei $100^{\circ}$ getrocknet) lieferten nach dem Glühen mit Kalk $0.2148 g$ Chlorsiber.

II. $0.1457 \mathrm{~g}$ Substanz gaben $0.0598 \mathrm{~g}$ Asche (Gold).

Aus einer anderen Darstellung:

III. $0.0740 \mathrm{~g}$ Substanz (bei $100^{\circ}$ konstant) gaben $0.0308 \mathrm{~g}$ Asche.

In 100 'Teilen:

\begin{tabular}{|c|c|c|c|}
\hline \multicolumn{3}{|c|}{ Gefunden } & \multirow{2}{*}{$\begin{array}{c}\text { Berechnet für } \\
\mathrm{C}_{5} \mathrm{H}_{4} \mathrm{~N}_{4} \mathrm{O} \mathrm{HClAu} \mathrm{Cl}{ }_{3}\end{array}$} \\
\hline I. & II. & III. & \\
\hline $\mathrm{C} 1 \ldots \ldots \ldots 30 \cdot 10$ & - & - & $30 \cdot 00$ \\
\hline $\mathrm{Au} \ldots \ldots \ldots-$ & $41 \cdot 05$ & $41 \cdot 62$ & $41 \cdot 42$ \\
\hline
\end{tabular}

Daß tatsächlich Hypoxanthin-Goldchlorid vorliegt, wurde weiters auch dadurch bewiesen, daß ein Teil des Goldsalzes mit Schwefelwasserstoff in der Kälte zerlegt und die filtrierte Lösung nach dem Neutralisieren mit Ammoniak eingedampft 
wurde. Die ausgeschiedene organische Substanz wurde getrocknet und auf den Zersetzungspunkt des Karnins $\left(230^{\circ}\right)$ geprüft; sie blieb jedoch bis $260^{\circ}$ ungefärbt. Schließlich lieferte die aus dem Golddoppelsalz abgeschiedene Base auch kein Acetylprodukt, während das Karnin, wie später ausführlich beschrieben, ein solches entstehen läßt. Die goldchloridhaltige Mutterlauge vom Hypoxanthin-Goldchlorid wurde gleichfalls mit Schwefelwasserstoff von Gold befreit und hinterließ nach dem Filtrieren und Eindampfen einen schwach gelb gefärbten Sirup, der Fehling'sche Lösung reduzierte, ein weiterer Beweis dafür, daß eine Zersetzung des Karnins eingetreten war.

Es schien wohl möglich, daß beim Versetzen einer Lösung von salzsaurem Karnin mit Goldchlorid sich ein Karnin-Goldchlorid bildet, da hiebei sofort die Ausscheidung einer Verbindung eintritt; jedenfalls aber ist das Karnin-Goldchlorid so unbeständig, daß es sich in kurzer Zeit sichtlich verändert und in Hypoxanthin-Goldchlorid übergeht.

Auf die Analyse des gleichfalls dargestellten Platindoppelsalzes, welches im Aussehen mit den vorliegenden Beschreibungen wohl übereinstimmte, haben wir mit Rücksicht auf die vorstehenden Resultate verzichtet.

Dieser Empfindlichkeit gegen Säuren steht eine außerordentliche Unempfindlichkeit gegen Basen gegenüber. Es konnte das Karnin mit Ammoniak, Barythydrat oder Kalilauge gekocht werden, ohne daß ein Steigen im Stickstoffgehalt eintrat. Es wurden aus diesem Grunde Versuche angestellt, Verbindungen des Karnins mit Basen zu seiner Reinigung zu benützen; von denselben seien das Lithium-, Natrium- und Baryumsalz erwähnt, welche äußerst zerfließlich sind und nur mit großen Verlusten umkristallisiert werden können. Sie sind wohl in Alkohol unlöslich, konnten aber trotzdem nicht zur Reinigung des Karnins mit Vorteil verwendet werden.

\section{Inosin.}

Die früher angeführten Differenzen in den Analysenresultaten, die an den Proben aus verschiedenen Karninkristallisationen zu Tage traten, im Vereine mit der Beobachtung, daß bei Auflösung des Karnins in heißem Wasser sich ein Teil 
leichter löst als der Rest, veranlaßten uns, eine Reinigung des Karnins durch Extraktion mit kaltem Wasser zu versuchen.

$1 \mathrm{~g}$ Karnin wurde $\mathrm{zu}$ wiederholten Malen mit je $200 \mathrm{~cm}^{3}$ kalten Wassers verrieben und die filtrierte Lösung eingedampft. Bei dem Konzentrieren schied sich nur eine sehr geringe Menge eines schwer löslichen Körpers am Rande der Flüssigkeit in Form dünner, matt weißer Linien aus, während eine größere Menge eines leicht löslichen Stoffes beim Verdunsten der letzten Wasserreste zu einer durchscheinenden, strahligkristallinischen Masse erstarrt. Diese konnte aus Alkohol umkristallisiert werden und zeigte den unscharfen Schmelzpunkt von $215^{\circ}$ unter gasiger Zersetzung und darauffolgender Braunund Schwarzfärbung, die bei $220^{\circ}$ vollender ist. Mit Rücksicht darauf, daß die Zusammensetzung der neuen Substanz es als wahrscheinlich erscheinen läßt, daß sie und die Inosinsäure aus der gleichen Muttersubstanz durch Abbau entstanden sein dürften, nennen wir dieselbe Inosin. Auch durch Methylalkohol kann die neue Substanz aus dem Karnin extrahiert werden; doch gelang es nach keinem der beiden Verfahren, das Produkt völlig frei von dem schwer löslichen Hypoxanthin zu erhalten. Dagegen fanden wir in der

\section{Acetylierung des Karnins}

eine aușgezeichnete Methode zur Abtrennung des Inosins aus dem Karnin.

$1 g$ Karnin wurde unter Zugabe eines Körnchens Natriumacetat mit Essigsäureanhydrid einmal aufgekocht und letzteres dann im Vakuum abdestilliert. Der Rückstand wurde mit Chloroform extrahiert, das Ungelöste nochmals in gleicher Weise acetyliert und wieder mit Chloroform extrahiert. Die Chloroformextrakte wurden vereinigt und 24 Stunden stehen gelassen, damit die Spuren von Hypoxanthin, welche in Lösung gegangen waren oder in der Flüssigkeit suspendiert blieben, Gelegenheit hatten, sich vollständig abzuscheiden. Es wurde sodann filtriert, bis zur Trocknis abdestilliert und aus absolutem Alkohol unter Anwendung von Tierkohle umkristallisiert. Die Substanz schied sich aus der alkoholischen Lösung beim Erkalten in prächtigen, seidenglänzenden Nadeln aus, die 
sofort rein waren und selbst nach viermaligem Umkristallisieren ihren Schmelzpunkt nicht änderten. Der Schmelzpunkt liegt bei $236^{\circ}$, ist jedoch unscharf, da bei dieser Temperatur Braunfärbung eintritt unter beginnender Zersetzung. Die Substanz ist in der etwa 50 fachen Menge absoluten Alkohols löslich und fällt beim Abkühlen fast vollständig heraus. War die Lösung des Acetylproduktes sehr verdünnt und erfolgte das Abkühlen langsam, so schied sich dasselbe in diamantglänzenden Kristallen, die zuweilen zu Drusen vereinigt waren, am Boden des Gefäßes ab. In Chloroform ist die Substanz sehr leicht, in Tetrachlorkohlenstoff dagegen sehr schwer löslich.

Die Analysen stimmen annähernd auf ein Triacetylinosin:

I. $0.1064 \mathrm{~g}$ der bei $100^{\circ}$ getrockneten Substanz gaben $14.0 \mathrm{~cm}^{3}$ feuchten Stickstoff bei $20^{\circ}$ und $732 \mathrm{~mm}$ Druck.

Iĩ. $0 \cdot 1010 \mathrm{~g}$ der bei $100^{\circ}$ getrockneten Substanz gaben $13 \cdot 1 \mathrm{~cm}^{3}$ feuchten Stickstoff bei $20^{\circ}$ und $739 \mathrm{~mm}$ Druck.

III. $0.2755 \mathrm{~g}$ Substanz, bei $100^{\circ}$ konstant, ergaben bei der Verbrennung $0.4970 \mathrm{~g}$ Kohlensäure und $0.1136 \mathrm{~g}$ Wasser.

IV. $0.1505 \mathrm{~g}$ Substan $z$ wurden mit $20 \mathrm{~cm}^{3}$ Barytlauge $\left(1 \mathrm{~cm}^{3}\right.$ enthielt $0.0099 \mathrm{~g}$ Baryumhydroxyd) eine Viertelstunde lang gekocht und mit 1/10 n.-Salzsäure unfer Anwendung von Lackmus als Indikator zurücktitriert, wobei $12.6 \mathrm{~cm}^{3}$ Salzsäure verbraucht wurden. Da ferner $20 \mathrm{~cm}^{3}$ Barythydrat zur Neutralisation $23.05 \mathrm{~cm}^{3}$ Salzsäure erforderten, waren von der durch Verseifung gebildeten Essigsäure $9 \cdot 1 \mathrm{~cm}^{3}$ Barytlauge verbraucht worden.

V. $0 \cdot 1541 \mathrm{~g}$ Substanz wurden nach der Methode des einen von uns der Acetylbestimmung unterworfen, wobei eine Essigsäuremenge abgespalten wurde, die zur Neutralisation $12.3 \mathrm{~cm}^{3} \quad 1 / 10 \mathrm{n}$.-Natronlauge verbrauchte. Schweflige Säure war bei der Bestimmung nicht aufgetreten.

In 100 Teilen:

\begin{tabular}{|c|c|c|c|c|c|}
\hline & \multicolumn{5}{|c|}{ Gefunden } \\
\hline & I. & II. & III. & IV. & V. \\
\hline$N \ldots \ldots \ldots \ldots$ & $.14 \cdot 53$ & $14 \cdot 49$ & - & - & - \\
\hline$C \ldots \ldots \ldots$ & - - & - & $49 \cdot 21$ & - & - \\
\hline$H \ldots \ldots \ldots \ldots$ & - & - & $4 \cdot 57$ & - & $\ldots$ \\
\hline \multirow[t]{3}{*}{$\mathrm{COCH}_{3} \ldots \ldots$} & - - & 一 & - & $35 \cdot 51$ & $34 \cdot 33$ \\
\hline & \multicolumn{5}{|c|}{ Berechnet für } \\
\hline & \multicolumn{3}{|c|}{$\mathrm{C}_{10} \mathrm{H}_{9} \mathrm{~N}_{4} \mathrm{O}_{5}\left(\mathrm{COCH}_{3}\right)_{3}$} & \multicolumn{2}{|c|}{$\mathrm{C}_{20} \mathrm{H}_{16} \mathrm{~N}_{8} \mathrm{O}_{9}\left(\mathrm{COCH}_{3}\right)_{6}$} \\
\hline$\ldots \ldots \ldots$ & \multicolumn{3}{|c|}{$14 \cdot 21$} & \multicolumn{2}{|c|}{$14 \cdot 54$} \\
\hline & \multicolumn{3}{|c|}{$48 \cdot 73$} & \multicolumn{2}{|c|}{$49 \cdot 87$} \\
\hline & \multicolumn{2}{|c|}{$4 \cdot 56$} & & \\
\hline $\mathrm{COCH}_{3} \ldots \ldots$ & \multicolumn{3}{|c|}{$32 \cdot 74$} & \multicolumn{2}{|c|}{$33 \cdot 50$} \\
\hline
\end{tabular}


Wir versuchten auch noch andere Derivate des Karnins darzustellen, um ein reines Produkt durch diesen Umweg zu gewinnen, doch zeigten alle diese Verbindungen, so z. B. die durch Einwirkung von Chlorkohlensäureester und Benzoylchlorid erhaltenen, so wenig erfreuliche Eigenschaften, daß wir auf deren Darstellung in größeren Mengen vorläufig verzichteten.

\section{Verseifung des Acetylinosins.}

$0.4085 \mathrm{~g}$ Acetylinosin wurden mit $100 \mathrm{~cm}^{3}$ 1/10 n.-Ätzbaryt eine halbe Stunde lang gekocht; sodann wurde mit der entsprechenden Menge 1/10 n.-Schwefelsäure der Baryt eben heraustitriert, so daß die Lösung weder baryt- noch schwefelsäurehaltig war. Da die Flüssigkeit infolge ihres Gehaltes an frei gewordener Essigsäure saure Reaktion zeigte, wurde der größte Teil derselben im Vakuum abdestilliert, der Rest aber über Schwefelsäure im Vakuum eindunsten gelassen. Hiebei erstarrte das Ganze zu einer Kristallmasse, die nach dem Abpressen auf einer Tonplatte ein Gewicht von $0.2675 g$ hatte, während die angewandte Menge des Acetylproduktes $0.2778 \mathrm{~g}$ Inosin liefern sollte. Das Rohprodukt wurde zunächst aus Wasser unter Anwendung von Tierkohle umkristallisiert, wobei aus verdünnter Lösung feine, seidenglänzende Nadeln, aus konzentrierter, sirupüser Lösung warzenförmige Drusen sich ausschieden. Als bestes Lösungsmittel zum Umkristallisieren erwies sich bisher 80 prozentiger Weingeist, aus dem das Inosin beim Erkalten in feinen, seidenglänzenden Nadein fast quantitativ ausfäl1t. In Wasser ist das Inosin wesentlich leichter löslich als das Karnin und das Hypoxanthin. Die Löslichkeit desselben in Wasser wurde bei $20^{\circ} \mathrm{C}$. bestimmt, indem aus der mit Kristallen völlig durchsetzten Flüssigkeit nach 24 stündigem Stehen bei dieser Temperatur $10 \mathrm{~cm}^{3}$ herausgenommen und abgedunstet wurden. Es blieb ein Rückstand von $0.1615 \mathrm{~g}$. In $100 \mathrm{~cm}^{3}$ der bei $20^{\circ}$ gesättigten wässerigen Lösung sind somit $1.615 \mathrm{~g}$ Inosin enthalten. Die Löslichkeit desselben ist daher mehr als zehnmal so groß als die des Hypoxanthins.

Auch dieses durch Verseifung des Acetylproduktes erhaltene, wie die folgenden Analysen beweisen, vollständig 
reine Inosin zeigt den unscharfen Schmelzpunkt von 215\%, bei welcher Temperatur bereits Zersetzung beginnt.

I. $0 \cdot 1965 \mathrm{~g}$ der bei $100^{\circ}$ getrockneten Substanz gaben bei der Verbrennung $0.3215 \mathrm{~g}$ Kohlensäure und $0.0817 \mathrm{~g}$ Wasser.

II. $0 \cdot 1028 \mathrm{~g}$ Substanz gaben bei. $18^{\circ} \mathrm{C}$. und $733 \mathrm{~mm}$ Barometerstand $19 \cdot 4 \mathrm{~cm}^{3}$ feuchten Stickstoff.

In 100 Teilen:

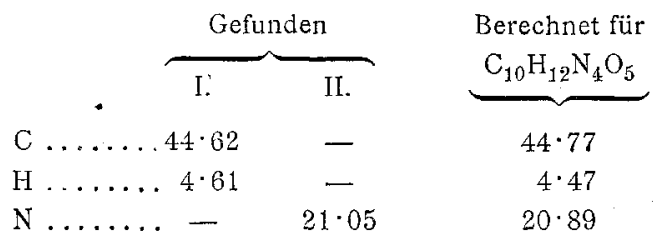

Bei Zusatz von Silbernitrat gestehen wässerige Inosinlösungen zu einer durchsichtigen Gallerte; das entstandene Silbersalz ist jedoch vollständig löslich in Ammoniak und dieser Umstand erklärt, warum manche Forscher das Karnin auch nicht in Spuren aufgefunden haben. Wenn infolge starken Salzsäuregehaltes der nach dem Zerlegen der in heißem Wasser löslichen Bleiverbindungen durch Schwefelwasserstoff erhaltenen Flüssigkeit beim Einengen Karnin nicht kristallisiert, schreibt Weidel vor, das Karnin nunmehr als Silbersalz zu fällen und das ausgeschiedene Karninsilber durch Waschen mit Ammoniak vom mitgefallenen Chlorsilber $z u$ befreien. Da wir nunmehr wissen, daß Inosinsilber in Ammoniak ebenso leicht löslich ist wie das Chlorsilber und nur das Hypoxanthinsilber in diesem Lösungsmittel unlöslich ist, wird es von dem mehr oder weniger schlechten Auswaschen mit Ammoniak abhängen, ob nach dem Zerlegen mit Schwefelwasserstoff eine größere oder geringere Menge Karnin oder ob überhaupt nur Hypoxanthin auskristallisiert. In der Tat hat auch Micko, ${ }^{1}$ ein geübter Analytiker, stets nur Hypoxanthin und nicht die Spur von Karnin aus Fleischextrakt erhalten.

1 Zeitschr. für Untersuchung der Nahrungs- und Genußmittel, 8, 231 und 10,393 . 


\section{Karnin als Gemenge.}

In wiederholten Versuchen wurde bei der Acetylierung von $1 \mathrm{~g}$ Karnin stets etwas mehr als $0.9 \mathrm{~g}$ Acetylinosin durch Extraktion mit Chloroform gewonnen, während dem chloroformunlöslichen Rückstande durch Behandeln mit kaltem Wasser das zugesetzte Natriumacetat und nicht ganz $0.1 \mathrm{~g}$ eines sirupösen Stoffes entzogen wurden und etwas über $0.3 \mathrm{~g}$ Hypoxanthin zurückblieben. Da nun $0.9 \mathrm{~g}$ Acetylinosin zirka $0.6 \mathrm{~g}$. Inosin entsprechen, ergibt sich die Zusammensetzung des Karnins wie folgt:

$\begin{array}{lccc} & \text { In } 1 g \text { Karnin } & \text { Molekulargewicht } & \text { Mole } \\ \text { Inosin } \ldots \ldots \ldots \ldots & 0.6 g & 268 & 1 \\ \text { Hypoxanthin..... } & 0.3 g & 136 & 1\end{array}$

Es stehen also die Mengen, in denen sich Inosin und Hypoxanthin aus Karnin isolieren lassen, ungefähr im Verhältnisse der Molekulargewichte dieser Stoffe. Es scheint somit das Karnin ein äquimolekulares Gemisch von Inosin und Hypoxanthin zu sein. Tatsächlich stimmen auch einzelne unserer früher angeführten Karninanalysen recht gut für ein solches Gemisch, wie z. B.:

In 100 Teilen:

\begin{tabular}{|c|c|c|c|c|}
\hline & \multicolumn{3}{|c|}{ Gefunden } & \multirow{2}{*}{$\begin{array}{c}\text { Berechnet für } \\
\mathrm{C}_{10} \mathrm{H}_{12} \mathrm{~N}_{4} \mathrm{O}_{5}+\mathrm{C}_{5} \mathrm{H}_{4} \mathrm{~N}_{4} \mathrm{O}\end{array}$} \\
\hline & I. & II. & III. & \\
\hline$C \ldots \ldots \ldots \ldots$ & - & $44 \cdot 14$ & - & $44 \cdot 55$ \\
\hline$H \ldots \ldots \ldots \ldots$ & - & $3 \cdot 90$ & - & $3 \cdot 96$ \\
\hline $\mathrm{N} \ldots \ldots \ldots \ldots 2$ & $27 \cdot 70$ & - & $27 \cdot 71$ & $27 \cdot 72$ \\
\hline
\end{tabular}

Die Abweichungen in den anderen Analysen erklären sich nunmehr auch in einfacher Weise, indem eben beim Waschen des Karnins mit Wasser demselben ein Teil des Inosins entzogen wird, wodurch der Stickstoffgehalt steigt.

\section{Optisches Drehungsvermögen des Inosins.}

Vor längerer Zeit schon haben wir die optische Aktivität von Inosinsäure wie auch von Karnin festgestellt. Da sich nun inzwischen herausstellte, daß das Karnin ein Gemenge ist und 
kaum von völlig konstanter Zusammensetzung erhalten werden kann, verzichten wir auf die Anführung der diesbezüglichen Resultate und wollen nur die am Inosin ausgeführte Bestimmung mitteilen.

$1.800 \mathrm{~g}$ Inosin wurden in Wasser gelöst und bei $18^{\circ} \mathrm{C}$. auf ein Volumen von $20 \mathrm{~cm}^{3}$ verdünnt. Diese Lösung ergab bei gleicher Temperatur und unter Verwendung von Natriumlicht

im $22 \mathrm{~cm}$-Rohr eine Drehung von $-9^{\circ} 45^{\prime}$

im $20 \mathrm{~cm}$-Rohr eine solche von $-8^{\circ} 53^{\prime}$

Daraus ergibt sich das spezifische Drehungsvermögen zu

$$
[\alpha]_{D}=-49 \cdot 2^{\circ}
$$

und das molekulare Drehungsvermögen zu

$$
[M]_{D}=-131 \cdot 8^{\circ} .
$$

\section{Molekulargewichtsbestimmungen.}

Da das Inosin mit Ausnahme von Wasser in keinem Lösungsmittel eine genügende Löslichkeit besitzt und die Konstanten für Wasser sehr kleine Werte haben, wollten wir uns mit der Bestimmung des Molekulargewichtes des Acetylinosins begnügen. Dieselbe wurde nach der Siedemethode in Beckmann's Apparat für strömenden Dampf ausgeführt.

Substanz: $0 \cdot 5345 \mathrm{~g}$ Acetylinosin, Lösungsmittel: Chloroform.

$\begin{array}{ccccc}\begin{array}{c}\text { Siedepunkts- } \\ \text { erhöhung }\end{array} & \text { Volumen } & \begin{array}{c}\text { Gewicht des } \\ \text { Lösungsmittels }\end{array} & \begin{array}{c}\text { Molekular- } \\ \text { gewicht }\end{array} \\ 0.15^{\circ} & 11.25 \mathrm{~cm}^{3} & 17 \cdot 17 \mathrm{~g} & 745 & 738 \\ 0.14^{\circ} & 12.50 \mathrm{~cm}^{3} & 19.07 \mathrm{~g} & 731 & \end{array}$

Dieses hohe Molekulargewicht entspricht nun allerdings keineswegs unseren Annahmen bezüglich der Molekulargröße des Inosins und seines Acetylderivates $\mathrm{C}_{10} \mathrm{H}_{9} \mathrm{~N}_{4} \mathrm{O}_{5}\left(\mathrm{COCH}_{3}\right)_{3}$, welches die Zahl 394 erfordern würde. Und doch erschien das obige Resultat durchaus nicht so unmöglich, da einerseits der Schmelzpunkt des Acetylinosins höher liegt als der des Inosins, während im allgemeinen bei Glykosiden durch Acetylierung derselbe stark herabgedrückt wird und da andrerseits 
die Resultate der Elementaranalyse und Acetylbestimmungen besser auf ein Produkt stimmen würden, das einer unter Wasseraustritt erfolgten Molekülverdopplung seine Entstehung verdankt, als auf das Triacetylinosin. Diesem Acetylinosin würde die Formel $\mathrm{C}_{32} \mathrm{H}_{34} \mathrm{~N}_{8} \mathrm{O}_{15}$ und das Molekulargewicht 770 zukommen. Daß die Übereinstimmung in den vorn angeführten Analysenresultaten mit den theoretischen Werten keine bessere ist, dürfte wohl darauf zurückzuführen sein, daß die Substanz. offenbar nur äußerst schwer rein zu erhalten ist, indem sie auch bei sorgfältigster Behandlung nach dem Umkristallisieren aus Alkohol und Trocknen im Vakuum nach Essigsäure riecht. Wir hoffen, daß es uns später noch gelingen wird, diese Verhältnisse aufzuklären.

Zunächst aber mußten wir uns doch entschließen, eine Molekulargewichtsbestimmung des Inosins selbst in wässeriger Lösung auszuführen. Auch hier kam Beckmann's Siedeapparat für strömenden Dampf zur Anwendung.

Substanz: $1 \cdot 1800 \mathrm{~g}$ Inosin, Lösungsnittel: Wasser.

$\begin{array}{cccc}\begin{array}{c}\text { Siedepunkts- } \\ \text { erhöhung }\end{array} & \begin{array}{c}\text { Volumen } \\ \text { der Lösung }\end{array} & \text { Molekulargewicht } & \text { Mittel } \\ 0.38^{\circ} & 5 \cdot 5 \mathrm{~cm}^{3} & 303 & \\ 0 \cdot 25 & 8 \cdot 5 & 302 & \\ 0 \cdot 20 & 10 \cdot 2 & 286 & 294 \\ 0 \cdot 19 & 11 \cdot 3 & 296 & \end{array}$

Der gefundene Mittelwert 294 beweist, daß dem Inosin die einfache Formel $\mathrm{C}_{10} \mathrm{H}_{12} \mathrm{~N}_{4} \mathrm{O}_{5}$ und das Molekulargewicht 268 zukommt. Wohl wäre auch an eine weitgehende elektrolytische Spaltung eines Doppelmoleküls zu denken, da das Inosin Salze zu bilden vermag, doch ist die Möglichkeit einer solchen bei der amphoteren Natur des Inosins äußerst unwahrscheinlich.

\section{Spaltung des Inosins.}

Da durch die vorstehenden Untersuchungen nachgewiesen ist, daß im Karnin dem Inosin Hypoxanthin beigemischt ist, war es notwendig, festzustellen, daß das Hypoxanthin auch als Spaltungsprodukt des Inosins auftritt. 
$0.2005 \mathrm{~g}$ Inosin wurden mit $50 \mathrm{~cm}^{3} 1 / 10$ n.-Schwefelsäure eine Stunde lang gekocht, hierauf wurde mit der berechneten Menge Barythydrat die Schwefelsäure entfernt, die Lösung zur Trocknis eingedampft und der Rückstand mit möglichst wenig Eiswasser extrahiert. Das Unlösliche (Hypoxanthin) wurde auf tariertem Filter gesammelt, bei $110^{\circ}$ getrocknet und gewogen. Die durch Eiswasser erhaltene Lösung wurde eingedampft, im Vakuum der Ölpumpe getrocknet, wobei ein Sirup zurückblieb, der gleichfalls gewogen wurde. Es wurden erhalten:

Unlösliches $0 \cdot 0940$, berechnet für Hypoxanthin $0 \cdot 1017$

Sirup $\quad 0.1135$, berechnet für eine Pentose 0.1122

Daß das in Wasser schwer lösliche Spaltprodukt des Inosins tatsächlich Hypoxanthin ist, geht aus seinen Eigenschaften, den Eigenschaften seines Chlorhydrates sowie aus den folgenden Analysen hervor:

I. $0.0960 \mathrm{~g}$ Substanz gaben bei $17^{\circ}$ und $738 \mathrm{~mm}$ Barometerstand $35.2 \mathrm{~cm}^{3}$ feuchten Stickstoff.

II. $0.2610 \mathrm{~g}$ Substanz, bei $110^{\circ}$ getrocknet, lieferten $0.4220 \mathrm{~g}$ Kohlensäure und $0.0740 \mathrm{~g}$ Wasser.

In 100 Teilen:

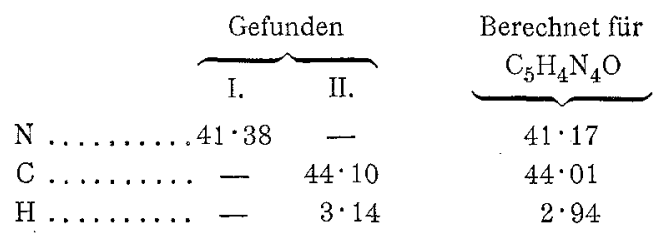

Der als zweites Spaltungsprodukt erhaltene Sirup schmeckt sehr süß und reduziert Fehling'sche Lösung beim Kochen; er konnte trotz vielfacher Bemühungen bisher nicht kristallisiert erhalten werden. Doch gelang die

\section{Darste1lung eines Osazons.}

$0.1980 \mathrm{~g}$ Sirup wurden 2 Stunden lang in wässeriger Lösung mit einem Überschusse von essigsaurem Phenylhydrazin im Wasserbad erhitzt. Schon in der Wärme, in reichlicherer Menge aber beim Erkalten scheiden sich dunkelgelb gefärbte Kristallnadeln aus; dieselben wurden mit Wasser 
gewaschen und aus 20 prozentigem Weingeist umkristallisiert, wobei hellgelbe, mikroskopische Nädelchen erhalten wurden. Die Ausbeute betrug $0.200 \mathrm{~g}$. Dieselben schmelzen bei $163^{\circ}$ unter Braunfärbung und Zersetzung.

$0.1200 \mathrm{~g}$ gaben bei $18^{\circ}$ und $739 \mathrm{~mm}$ Barometerstand $17.8 \mathrm{~cm}^{3}$ feuchten Stickstoff.

In 100 Teilen:

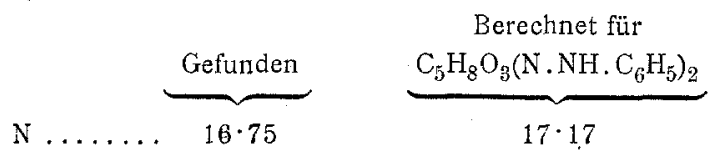

Da ebenso die Reaktionen mittels Phloroglucin und Orcin auf eine Pentose positiv ausfielen, so ist somit nachgewiesen, da $\beta$ das Inosin ein Pentosid ist und daß es beim Erwärmen mit Säuren leicht in Hypoxanthin und eine Pentose zerfällt. Die Konfiguration dieser Pentose festzustellen, das muß einer späteren Mitteilung vorbehalten bleiben.

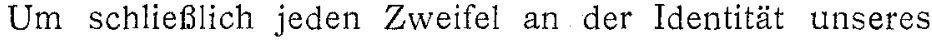
Karnins mit Weidel's Karnin auszuschließen, wurde noch ein Präparat zum Vergleiche herangezogen, das sich in der Sammlung des inzwischen verstorbenen Herrn Prof. Hugo Weidel vorfand und das uns in liebenswürdigster Weise durch Herrn Prof. R. Wegscheider zur Verfügung gestellt wurde. Obgleich die Menge desselben äußerst gering war, so konnte doch durch eine Analyse und den Schmelzpunkt zweier Körper, die daraus dargestellt wurden, die Identität der beiden als Karnin bezeichneten Gemische nachgewiesen werden.

Einmal ließ sich aus diesem Präparat das Inosin mit dem Schmelzpunkte $215^{\circ}$ durch einfache Extraktion mit Methylalkohol gewinnen. Ferner wurden $0.3 \mathrm{~g}$ davon nach der oben angeführten Methode acetyliett und $0.27 g$ in Chloroform lösliches Acetylprodukt erhalten, das, aus Alkohol umkristallisiert, den Schmelzpunkt $236^{\circ}$ zeigte. $0.1 \mathrm{~g}$ blieb als in Chloroform unlöslicher Rückstand zurück. Das Acetylprodukt wurde sodann mit Barytlauge auf gleiche Weise wie früher verseift 
und eine Menge Inosin gewonnen, die nach dem Umkristallisieren aus Weingeist eben noch für eine Stickstoff bestimmung reichte. Der Schmelzpunkt wurde dem früher erhaltenen $\left(215^{\circ}\right.$ unscharf) gleich gefunden.

$0.0714 \mathrm{~g}$ Substanz gaben bei $21^{\circ}$ und $737 \mathrm{~mm}$ Barometerstand $13.7 \mathrm{~cm}^{3}$ feuchten Stickstoff.

In 100 Teilen:

$\underbrace{\text { Gefunden }}_{21 \cdot 22} \quad \underbrace{\begin{array}{c}\text { Berechnet für } \\ \mathrm{C}_{10} \mathrm{H}_{12} \mathrm{~N}_{4} \mathrm{O}_{5}\end{array}}_{20 \cdot 89}$

